\title{
Demokratische Legitimation in der Europäischen Union
}

von Peter M. Huber

\begin{abstract}
Die Lissabon-Entscheidung des Bundesverfassungsgerichts führte zu einer Intensivierung der Diskussion um die Auswirkungen und die Entwicklungsfähigkeit des grundgesetzlich normierten Demokratieprinzips. Im Rahmen dieser Diskussion befasst sich der vorliegende Beitrag mit der Frage nach der demokratischen Legitimation im Verhältnis von Europäischer Union, Nationalstaat und substaatlicher Ebene. Ausgehend von der Beobachtung, dass die Wahlen zum Europäischen Parlament nur eine geringe Wählermobilisierung zeitigen und auf Grundlage einer vergleichenden Betrachtung der demokratischen Praxis in unterschiedlichen europäischen Staaten werden unter Verweis auf die zentrale Rolle des Rats der Europäischen Union einige bedeutsame Eigenheiten des europäischen Mehr-Ebenen-System herausgearbeitet, um anschließend die Rolle der EU als wechselseitige Auffang- und Kooperationsordung zu beschreiben. Als Reformoption wird neben der besseren Einbindung nationaler Legislativen in den europapolitische Prozess v. a. hinsichtlich der begrenzten Legitimationskraft des Europäischen Parlaments - auf das Desiderat politischer Parteien auf unionaler Ebene verwiesen.
\end{abstract}

The German Constitutional Court's decision on the Treaty of Lisbon led to an intensified debate on the effects of the Basic Law's principle of democracy and its potential for further development. In the context of this debate, the article seeks to discuss the issue of democratic legitimisation within theEU-system, its Member States and sub-state entities. Based on the observation that elections to the European Parliament largely fail to mobilize voters and supported by a comparative overview over democratic practices in several European states, the central role of the Council is used to display some important particularities of a multi-level-system. This leads to a description of the EU as a reciprocal order of support and co-operation. In conclusion, both the better integration of national legislatures into the process of European policy-making and - not least in view of lacking legitimising powers to the European Parliament - political parties at the Union level are seen as a potential option for further reform and development.

\section{Geringe Akzeptanz der Europawahlen}

Nicht nur wegen der sehr geringen Wahlbeteiligung sind die Europawahlen des Jahres 2009 und ihr Beitrag zum demokratischen Legitimationsniveau der Europäischen Union in der Wahrnehmung der Öffentlichkeit (wiederum) deutlich hinter dem zurück geblieben, was Lehrbücher und Kommentare in idealisieren- 
der Weise regelmäßig mit ihnen verbinden. Dabei hat es nicht an Versuchen gefehlt, die Wahlbeteiligung zu stärken. Zum „Plebiszit“ über den Vertrag von Lissabon wurden sie ausgerufen, in Nordrhein-Westfalen mit den Kommunalwahlen zusammengelegt, weil dies, so die Annahme, die Wahlbeteiligung höher ausfallen lassen würde, ${ }^{1}$ und in Dänemark wurde - um ein drittes Beispiel zu nennen - das Referendum über die weibliche Thronfolge mit den Europawahlen zusammengelegt, um das Volk an die Urnen zu bringen.

An dem unbefriedigenden Ergebnis hat sich seit 1978 jedoch nichts verändert. Sicher, seitdem sind die Verträge von Maastricht (1992/93), Amsterdam (1997/ 99), Nizza (2000/01) und Lissabon (2009) in Kraft getreten. Mit all diesen Verträgen sind die Befugnisse des Europäischen Parlaments ausgeweitet worden, so dass das sog. Mitentscheidungsverfahren heute die Regel ist. Gleichwohl stand Herr Pöttering, der seinerzeitige Präsident des Europäischen Parlaments, am Abend der Europawahlen frustriert vor den Fernsehkameras und beklagte sich darüber, dass die Leute nicht verstünden, wie wichtig Europa sei. Dabei zog er seinen Fühlfederhalter heraus, hielt ihn hoch und erklärte, dass er mit ihm das Klimapaket unterschrieben habe. Dennoch interessiere sich das Volk nicht für das Europäische Parlament.

Die geringe Wahlbeteiligung bei den Europawahlen ist freilich weniger Ausdruck einer dezidierten Ablehnung des Europäischen Parlamentes ist, sondern, was vielleicht schlimmer ist, von Desinteresse. Zwar gibt es auch im Europäischen Parlament Vorgänge, die sich durchaus für eine Skandalisierung eignen würden: Die "schwarze Liste“ von parlamentskritischen Journalisten, die mit Hausverbot belegt werden, die Forderung, Gelder für die zusätzliche Altersversorgung der Abgeordneten, die in Anleihen der insolvent gewordenen Bank Lehman Bros. investiert waren, aus dem EU-Budget zu ersetzen. Das sind zwar, wie der Blick auf die aktuellen Spesenskandale in Großbritannien zeigt, keine Spezifika Europas oder des Europäischen Parlaments; spezifisch ist jedoch, dass derartige Vorgänge gar nicht das Potential für einen Skandal zu besitzen scheinen.

Grund dafür ist, dass die Vorstellung davon, welche Rolle das Europäische Parlament in der institutionellen Architektur Europas spielen soll, nicht hinreichend klar ist, bzw. jeder insoweit andere Vorstellungen, (Vor-)Verständnisse und Erwartungshaltungen hat. Deshalb ist es bei der Beurteilung des Beitrags, den

1 VerfGH NRW, Urt. v. 18. 02. 2009, 24/08 (Zusammenlegung von Kommunal- und Europawahlen), abgedruckt in: Deutsches Verwaltungsblatt, 124/8 (2009), $516 \mathrm{ff}$. 
das Europäische Parlament zur demokratischen Legitimation der EU leistet, wie bei dem sprichwörtlichen Glas Wasser, bei dem man endlos darüber debattieren kann, ob es halb voll oder halb leer ist. $\mathrm{Zu}$ welchem Ergebnis man gelangt, hängt vom Blickwinkel ab.

Passend zum Thema findet sich in der Juni-Ausgabe des Spiegel ein Interview mit Timothy Gordon Ash, in dem er ebenfalls auf die Europawahlen eingeht. ${ }^{2}$ Die Redakteure hatten ihn sinngemäß gefragt, ob die vergangenen Europawahlen nicht gezeigt hätten, dass es hier in Wahrheit gar nicht um Europa gehe, sondern um nationale Politik, und dass Europa gar nicht vereint, sondern zersplittert sei. Herr Ash antwortete darauf mit der Bemerkung, dass die eigentlichen Europawahlen Ende September in Deutschland stattfänden. Denn für die Zukunft der Europäischen Union sei die Bundestagswahl wichtiger als die Europawahlen. Daran habe der Kompetenzzuwachs des Europäischen Parlaments und die Ausdehnung der Mitentscheidungsmöglichkeiten ebenso wenig etwas geändert wie der im Lissabonner Vertrag vorgesehene Bürgerantrag. Mit vermehrten Kompetenzen des Europäischen Parlaments allein, das ist wohl die These, werden die Europawahlen noch nicht zu jenem Integrationsvorgang, den Rudolf Smend im Auge gehabt hatte. ${ }^{3}$ Und in der Tat wird man schwerlich behaupten können, dass sich die Bürger der Europäischen Union in den Europawahlen zu einer Einheit verbinden und finden.

$\mathrm{Ob}$ das ein demokratierechtliches Problem ist, darüber gehen die Meinungen auseinander, schon weil es sehr heterogene Interpretationsansätze für das Demokratieprinzip gibt. Wenn man sie nur offen, beliebig oder anspruchslos genug konzipiert, wird man freilich auch keine Probleme mit der demokratischen Legitimation im Mehr-Ebenen-System der EU haben.

\section{Gewährleistungsgehalt der Demokratiegebote}

Was genau das Demokratieprinzip fordert oder gewährleistet, ist nicht ohne weiteres festzustellen. Das gilt schon im Kontext des Nationalstaats, wo es jedenfalls in Deutschland - seit dem KPD-Urteil vom Bundesverfassungsgericht als voraussetzungsvolles, relativ anspruchsvolles und mit spezifischen Vorgaben

2 Der Spiegel v. 15.06. 2009.

3 Zur Integrationslehre Smend, R.: Integrationslehre, in: Handwörterbuch der Sozialwissenschaften, Bd. 5, Stuttgart, 1956, $299 \mathrm{ff}$; zu deren Bedeutung für Wahlen Badura, P.: Über Wahlen, in: Archiv des öffentlichen Rechts, 97/1 (1972), 1-11, hier 3. 
versehenes Verfassungsprinzip entfaltet worden ist $\mathrm{t}^{4}$ und wo seit der Wiedervereinigung, seit die Bundesrepublik Deutschland zu einem mehr oder weniger in sich ruhenden und „normalen“ Nationalstaat herangewachsen ist, intensiv über Demokratie diskutiert wird. Seither stellen wir die Frage ob „Demokratie“ eine „Regel“ im rechtstheoretischen Sinne ist, oder ein optimierungsbedürftiges (Verfassungs-)Prinzip. Gleichzeit ist zunehmend unklar geworden, ob Demokratie um es mit Lincolns Gettysburgh Address zu sagen - wirklich das ,Government of the people, by the people and for the people" bedeutet und ob es insoweit wirklich auch auf den output von Entscheidungsstrukturen und -verfahren ankommt. Dem lässt sich jedenfalls entgegenhalten, dass ein auf Akzeptanz stoßender output, eine ,gute Politik“, nicht (nur) von der Legitimations- oder Regierungsform abhängt; auch Friedrich der Große hat - gemessen an den Maßstäben des 18. Jahrhunderts - eine verhältnismäßig ,gute Politik“ für seine Untertanen betrieben; eine Demokratie war Preußen deshalb nicht.

\section{Notwendigkeit einer Konturierung}

Was das Demokratieprinzip fordert oder gewährleistet, hat mit der methodischen Frage zu tun, was man alles in dieses Prinzip hinein interpretiert. Auch das Bundesverfassungsgericht lässt es insoweit an Klarheit fehlen, wenn es etwa den Grundsatz der Demokratie typischerweise mit dem Gedanken von Freiheit und Rechtsstaat verbindet. Die Aufgabe des Juristen besteht jedoch darin, Begriffe so $\mathrm{zu}$ fassen, dass sie zu anderen abgrenzbar sind, so dass unterschiedliche Begriffe und die hinter ihnen stehenden Wertentscheidungen im Falle einer Kollision rational miteinander abgewogen und in einen verhältnismäßigen Ausgleich gebracht werden können. Wenn man dagegen, wie es mitunter geschieht, alles Gute, Wahre und Schöne unter den Begriff der Demokratie zu fassen sucht, bleibt diese verfassungsrechtliche Grundentscheidung konturenlos und diffus. ${ }^{5}$

Seit der Entscheidung des Bundesverfassungsgerichts zu den Emschergenossenschaften ${ }^{6}$ kann als anerkannt gelten, dass es sich bei der verfassungsrechtlichen Grundentscheidung für die Demokratie um ein Prinzip im Rechtssinne handelt,

5 Huber, P.M.: Demokratie in Europa - Zusammenfassung und Ausblick, in: Bauer, H./Huber, P.M./Sommermann, K.-P. (Hg.): Demokratie in Europa, Tübingen, 2005, 491-512; mit Blick auf das Sozialstaatsprinzip ähnlich Volkmann, U.: Aussprache, in: Veröffentlichungen der Vereinigung der Deutschen Staatsrechtslehrer, 64 (2005), $91 \mathrm{ff}$.

BVerfGE 107, 59 ff. (Wasserverbände NRW), hier 91. 
d.h. um eine optimierungsbedürftige Grundentscheidung. Dies stößt in der Literatur zwar nicht nur auf Zustimmung; die Linie ist vom Bundesverfassungsgericht in den letzten Jahren jedoch - sieht man einmal von der Entscheidung zum Vertrag von Lissabon $\mathrm{ab}^{7}$ - so vorgezeichnet und ausgebaut worden.

\section{Der unionale Grundsatz der Demokratie}

Blickt man dagegen auf die europäische Ebene, dann findet die Demokratie natürlich auch dort eine rechtliche Verankerung. Im bisherigen Art. 6 EUVNizza und erst recht im Vertrag von Lissabon, der einen ganzen Abschnitt über die Demokratie und das demokratische Leben enthält (Art. 9 ff. EUV-Lissabon), ist davon die Rede, dass die Europäische Union bzw. ihre Arbeitsweise auf dem Grundsatz der (repräsentativen) Demokratie beruht. Daneben gibt es Wahlverfahren, Unionsbürgerschaft und andere Formen der Partizipation - vom Petitionsrecht bis zum Bürgerbeauftragten - die der demokratischen Mitwirkung der Bürgerinnen und Bürger dienen.

Trotzdem ist es keine große Überraschung, dass das unionale Demokratieprinzip nicht dieselbe konsistente Konzeption aufweist, wie sie für das nationalstaatliche Demokratieprinzip in Deutschland und anderen Mitgliedstaaten kennzeichnend ist. Es markiert vielmehr jenen Mindeststandard, wie er sich aus den Verfassungsordnungen der Mitgliedstaaten und der Europäischen Menschenrechtskonvention ableiten lässt und wie er vom Vertragsgeber kodifiziert worden ist.

\section{a) Kein übergreifendes Verfassungsprinzip}

Lässt man die Verfassungsordnungen der Mitgliedstaaten Revue passieren, zeigt sich zunächst, dass es in einer Reihe von ihnen überhaupt kein Demokratieprinzip i.e. S. gibt. Das gilt gerade auch für die Schweiz, die zwar nicht Mitglied der Europäischen Union ist, in Sachen Demokratie jedoch eine Art „benchmark“ darstellt. Wie andernorts auch gibt es dort lediglich Regeln, die das Wahlverfahren, die direkte Demokratie und Ähnliches betreffen, aber keine überwölbende Konzeption, kein bestimmtes Leitbild der Demokratie, das bei der Anwendung und Auslegung der einzelnen demokratierelevanten Bestimmungen zugrunde gelegt werden könnte. Überhaupt scheint man dort, wo die Partizipationsmög-

7 BVerfG, Urt. v. 30.06. 2009, 2 BvE 2/08 u. a. (Vertrag von Lissabon), abgedruckt in: Neue juristische Wochenschrift, 62/31 (2009), 2267-2295, hier Rz. 216. 
lichkeiten des Einzelnen besonders umfangreich sind, auf einen ideellen oder dogmatischen Überbau eher verzichten zu können.

Wenn es aber in einer erheblichen Zahl von Mitgliedstaaten, womöglich in ihrer Mehrheit, kein übergreifendes Prinzip der Demokratie gibt, dann hat dies notgedrungen auch für die Ebene der Europäischen Union Konsequenzen. Wenn der EUV pauschal erklärt, dass Europa auf dem Grundsatz der Demokratie beruhe und damit auf die Verfassungsordnungen der Mitgliedstaaten verweist, dann kann dies nicht in jenem anspruchsvoll-idealistischen Sinne verstanden werden, wie er für die aktuelle deutsche Diskussion kennzeichnend ist. Der unionale Grundsatz der Demokratie erschöpft sich vielmehr in den Verfahren und Institutionen, die dem Einzelnen Partizipation ermöglichen und zu seiner Selbstbestimmung beitragen. Das gilt namentlich für das Wahlrecht zum Europäischen Parlament, den Bürgerantrag, das Petitionsrecht, das Recht auf Anrufung des Bürgerbeauftragten sowie vergleichbarer Instrumente. Ob sich durch die nähere Ausgestaltung der demokratischen Grundsätze in den Art. 9 ff. EUV-Lissabon etwas ändern wird, lässt sich derzeit nicht absehen.

Ein Problem stellt in diesem Zusammenhang freilich die auf europäischer Ebene nicht gewährleistete Wahlrechtsgleichheit dar. Wie das Zusatzprotokoll zur EMRK, so sieht der EUV auch in Zukunft nur allgemeine, freie und unmittelbare, aber eben keine gleichen Wahlen vor (Art. 190 Abs. 4 EGV/Art. 223 Abs. 1 AEUV). Nach wie vor normiert der EGV und in Zukunft das Sekundärrecht einen ,ponderierten Schlüssel“ für der Repräsentation der im Wesentlichen nach Mitgliedstaaten gegliederten Unionsbürger durch das Europäische Parlament, der unter politisch-diplomatischem Blickwinkel zwar einleuchtet, aus rechtlicher Perspektive jedoch Zweifeln ausgesetzt ist.

\section{b) Überprüfungsbedürftigkeit des Status quo}

Abgesehen davon, dass der EUV in seiner Lissabonner Fassung bei den Bestimmungen über die demokratischen Grundsätze die Gleichheit aller Unionsbürgerinnen und Unionsbürger betont, denen ein gleiches $\mathrm{Ma} ß$ an Aufmerksamkeit zuteil werden müsse (Art. 9 Satz 1 EUV-Lissabon), hat der Europäische Gerichtshof für Menschenrechte (EGMR) Abstriche vom Grundsatz der Wahlrechtsgleichheit freilich immer kritisch gesehen. Er hat aus Art. 3 1.ZP EMRK i. V.m. Art. 14 EMRK (Diskriminierungsverbot) auch konkrete Anforderungen an die Größe von Wahlbezirken abgeleitet. Für die Europäische Union ist das zwar bisher noch nicht geschehen; berücksichtigt man jedoch, dass der Europäi- 
sche Gerichtshof für Menschenrechte seit seiner Entscheidung in Sachen Matthews ${ }^{8}$ die an die Mitgliedstaaten gerichteten wahlrechtlichen Anforderungen mittelbar auch auf die Europäische Union im Allgemeinen und das Europäische Parlament im Besonderen erstreckt, so reichen die Anforderungen an eine gleichheitskonforme Verteilung der Sitze im Straßburger Parlament bereits de lege lata womöglich weiter als es der bisherigen Unionspraxis entspricht. Dem kann und muss bei einer Anpassung der Sitzverteilung Rechnung getragen werden. Tritt die EU der EMRK auf der Basis des Vertrags von Lissabon bei (Art. 6 Abs. 2 Satz 1 EUV-Lissabon), so gilt das erst recht.

\section{Divergenz von nationaler und unionaler Ebene}

Was Demokratie auf europäischer Ebene bedeutet, ist weniger voraussetzungsvoll, weniger materiellrechtlich angeleitet oder aufgeladen, als es im nationalen Kontext der Fall ist. So stellt sich die Frage, ob der Umstand, dass der für demokratische Wahlen so elementare Grundsatz des ,, one man, one vote “ auf europäischer Ebene nur mit erheblichen Einschränkungen gilt - nicht was den Zähl-, wohl aber was den Erfolgswert der Stimmen angeht - für die demokratische Legitimation im Mehr-Ebenen-System nicht Konsequenzen haben muss. ${ }^{9}$

\section{Demokratie im Mehr-Ebenen-System}

Die Antwort auf diese Frage setzt zunächst die Einsicht voraus, dass Demokratie im „Mehr-Ebenen-System“ mit besonderen Problemen verbunden ist. Ein „Mehr-Ebenen-System“ - sei es Bundesstaat oder Staaten- und Verfassungsverbund - ist, anders als es manchmal dargestellt wird, ${ }^{10}$ - kein herrschaftsfreies Gebilde; auch im „Mehr-Ebenen-System“ gibt es ein Gravitationszentrum und auf dieses zielt das Erfordernis demokratischer Legitimation in erster Linie.

8 EGMR, Urt. v. 18.02.1999, Nr. 24833/94 (Matthews/Vereinigtes Königreich), abgedruckt in: Europäische Grundrechte-Zeitschrift, 26/7-8 (1999), $200 \mathrm{ff}$.

9 Zu diesem Gedanken BVerfG, Vertrag von Lissabon, a.a. O., Rz. 271; früher bereits Huber, P. M.: Die Rolle des Demokratieprinzips im europäischen Integrationsprozeß, in: Staatswissenschaften und Staatspraxis, 3 (1992), 349-378, hier $367 \mathrm{ff}$.

10 Pernice, I.: Europäisches und nationales Verfassungsecht, in: Veröffentlichungen der Vereinigung der Deutschen Staatsrechtslehrer, 60 (2001), $163 \mathrm{ff}$; Mayer, F. C.: Europäische Verfassungsgerichtsbarkeit, in: Bogdandy, A.v./Bast, J. (Hg.): Europäisches Verfassungsrecht, 2. Aufl., Berlin, 2009, 559-607, hier 595. 


\section{Unterschiede zwischen Bundesstaat und Staatenverbund}

Das gilt für den Bundesstaat genauso wie für die Europäische Union. Blickt man etwa auf die Landtagswahlen in Deutschland, ${ }^{11}$ dann erkennt man, dass sie ihre eigentliche Funktion, eine politische Präferenzentscheidung auf regionaler Ebene und nach Maßgabe regionaler Kriterien zu sein, zu einem erheblichen Teil eingebüßt haben. Das hängt zwar im Detail auch von den politischen Konstellationen auf Bundes- und Landesebene ab; es ist jedoch offenkundig, dass Landtagswahlen typischerweise auch, wenn nicht sogar in erster Linie Testwahlen für den Bund sind. Damit ist ihre Legitimationsfunktion für die Landesstaatsgewalt formal zwar gegeben; in der Sache aber ist sie doch durch erhebliche Erosionstendenzen gekennzeichnet, weil das politische Gravitationszentrum unseres Landes heute eben nicht in Düsseldorf, Erfurt oder München liegt, sondern in Berlin.

Eine Ebene höher - im Verhältnis zwischen Berlin und Brüssel oder Straßburg stellt sich das jedoch anders dar. Denn im Verhältnis zwischen der Europäischen Union und den Mitgliedstaaten haben wir es, anders als zwischen Bund und Ländern, nicht mit einem Verhältnis der Über- und Unterordnung zu tun. Vielmehr sind die Mitgliedstaaten, wie man in Anlehnung an die Rechtsprechung des Bundesverfassungsgerichts formulieren kann, Herren der Verträge. ${ }^{12}$ Die Kompetenz-Kompetenz liegt bei ihnen und deshalb bilden auch sie das Gravitationszentrum der politischen Entscheidungsprozesse. Das ist unter verfassungsrechtlich-dogmatischem Blickwinkel nahezu unstreitig, gilt bei zutreffender Betrachtungsweise aber auch aus der Perspektive des Unionsrechts. Auf Art. 6 EUV lässt sich insoweit ebenso verweisen wie auf die Art. 48 und 49 EUV, die Vorschriften über das System der Eigenmittel (Art. 269 EGV/Art. 311 AEUV) oder auf die Evolutivklauseln im Bereich der Unionsbürgerschaft (Art. 22 EGV/Art. 25 AEUV) und anderen Bereichen.

Die Fehleinschätzung, der Herr Pöttering am Wahlabend erlegen ist und die er mit vielen deutschen Europapolitikern und -rechtlern teilt, beruht wohl darauf, dass die Erfahrungen mit dem bundesstaatlichen „Mehr-Ebenen-System“ in Deutschland unbesehen auf die unionale Ebene übertragen werden, und dass man im Europäischen Parlament so etwas sieht oder sehen will wie den Deutschen

11 Die Föderalismusreformen der vergangenen Jahren haben da auch eine zentrale Rolle gespielt.

12 Etwas anderes gilt allenfalls aus dem Blickwinkel der Niederlande, die ihre Rechtsordnung auf der Grundlage eines monistischen Verständnisses dem acquis communautaire vollständig unterordnen, vgl. Wessels, R. A./van de Griendt, W. E.: Offene Staatlichkeit: Niederlande, in: Bogdandy, A.v./Cruz Villalón, P./Huber, P. M. (Hrsg.): Handbuch Ius publicum europaeum, Bd. 2, Heidelberg, 2008, § 19, Rn. $26 \mathrm{ff} ., 37 \mathrm{ff}$. 
Bundestag auf unionaler Ebene. Das aber ist er nicht. Denn das institutionelle Gefüge der Europäischen Union unterscheidet sich grundlegend von dem, das wir vom Nationalstaat, d.h. vom Bundesstaat in seiner spezifisch deutschen und unitarischen Prägung kennen.

Wenn man dies sine ira et studio zu Kenntnis nähme, würde sich manche Enttäuschung vermeiden lassen; zudem fiele die Beurteilung der Europawahlen weit weniger dramatisch aus als dies in der Öffentlichkeit und bei den betroffenen Politikern regelmäßig der Fall ist. Denn bei Lichte besehen gleicht das Europäische Parlament eher dem Reichstag des Kaiserreichs, der zwar auch in allgemeinen, freien und gleichen Wahlen (der Männer) gewählt wurde, aber dennoch nur zweite Kammer war. Zudem verfügt das Europäische Parlament nur über eine „negative demokratische Kompetenz"13, kann also Entscheidungen maximal verhindern, sie jedoch - von ganz wenigen Angelegenheiten abgesehen ${ }^{14}-$ nicht selbst initiieren. Es ist insoweit letztlich auf das Initiativmonopol der Kommission angewiesen oder auf die Aktivitäten des Rates.

\section{Der Rat als Gravitationszentrum der EU}

Politisches und rechtliches Gravitationszentrum der Europäischen Union ist der Rat, an dessen Entscheidungen das Europäische Parlament mehr oder weniger gleichberechtigt mitwirkt. Wenn dem so ist und wenn man erkennt, dass das Schwergewicht der politischen Entscheidungen in die Zuständigkeit des Rates fällt, dann hat das auch Konsequenzen für die demokratische Legitimation der Europäischen Union. Während sich im Gefüge des Grundgesetzes alles auf den Deutschen Bundestag konzentriert, er gewissermaßen die „Mitte der Demokratie" bildet, wie es Paul Kirchhof einmal treffend genannt hat, ${ }^{15}$ gilt dies für das Europäische Parlament nicht oder doch nur in sehr eingeschränktem Maße. Es hat kein eigenständiges Kreationsrecht bei der Einsetzung der Kommission oder bei der Wahl der Richter und es wirkt auch an der Gesetzgebung lediglich mit.

13 Huber, P. M.: Die Rolle des Demokratieprinzips, a. a. O., 358.

14 Siehe etwa das Wahlrecht oder das Abgeordnetenstatut: Art. 190 Abs. 4 und 5 EGV bzw. Art. 223 Abs. 1 und 2 AEUV.

15 Kirchhof, P.: Das Parlament als Mitte der Demokratie, in: Brenner, M. u.a. (Hg.): Der Staat des Grundgesetzes - Kontinuität und Wandel: Festschrift für Peter Badura zum siebzigsten Geburtstag, Tübingen, 2004, 237-262. 


\section{Die EU als wechselseitige Auffang- und Kooperationsordnung}

Betrachtet man nicht nur die Institutionen, sondern die Rechtsordnung der EU insgesamt, dann ist sie im Übrigen nicht jene selbstreferentielle, an Münchhausen erinnernde Rechtsordnung, die sich autopoietisch aus dem Nichts erschaffen hat. Diese politisch geschickt und erfolgreich propagierte These ${ }^{16}$ hält einer kritischen Überprüfung nicht stand.

\section{Die Verfassungen der Mitgliedstaaten als Grundlage der EU}

Die rechtliche Grundlage, auf der sich die europäische Integration vollzieht, sind die Verfassungen der Mitgliedstaaten oder - genauer - ihr europafester Identitätskern. Das entspricht der Rechtsprechung des Bundesverfassungsgerichts, expliziten verfassungsrechtlichen Vorgaben in zahlreichen Mitgliedstaaten von Schweden (Kap. $10 \S 5$ Schwed.Regeringsform) ${ }^{17}$ über Polen (Art. 8 Nr. 1 Poln.Verf.) bis nach Tschechien (Art. $9 \mathrm{f}$. Tschech.Verf.) und wird auch durch das Unionsrecht anerkannt - durch die zahlreichen Vorschriften, in denen es selbst ausdrücklich auf die Verfassungsordnung der Mitgliedstaaten verweist. Wenn aber schon der Wortlaut des Unionsrechts an die Verfassungsordnung der Mitgliedstaaten anknüpft, dann kann dieses für Auslegung und Anwendung nicht irrelevant sein. Im Gegenteil: nationales (Verfassungs-)Recht und Unionsrecht beeinflussen sich gegenseitig und bilden eine wechselseitige Auffangordnung.

Sieht man genauer hin, so erkennt man, dass auch der Europäische Gerichtshof dies nicht anders sieht. Wenn die Dworkin'sche These richtig ist, dass „Fitting

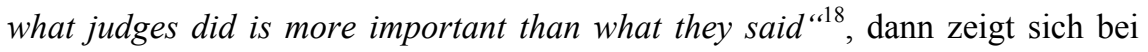
einer Musterung der Luxemburger Rechtsprechung, dass der Gerichtshof die integrationsfesten Kerne des nationalen Verfassungsrechts stets respektiert hat und mit den nationalen Verfassungs- und Obergerichten nicht wirklich im Streit liegt. Im Fall der Tanja Kreil, jener jungen Dame, die zur Bundeswehr wollte, dies aber wegen des Verbots in Art. 12a Abs. 4 Satz 2 GG a.F., wonach Frauen keinesfalls Dienst mit der Waffe leisten durften, nicht konnte, hat sich der Generalanwalt La Pergola intensiv mit den deutschen Grundgesetzkommentaren beschäftigt und mit der Interpretation unserer Ewigkeitsgarantie. Erst nachdem er

16 EuGH, Urt. v. 15.07. 1964, Rs. 6/64 (Costa v. Enel), Slg. 1964, 1141.

17 Nergelius, J.: Offene Staatlichkeit: Schweden, in: Bogdandy, A.v./Cruz Villalón, P./Huber, P.M. (Hrsg.): Handbuch Ius publicum europaeum, Bd. 2, Heidelberg, 2008, § 22 Rn. $11 \mathrm{ff}$.

18 Dworkin, R.: Law's Empire, London, 1986, $247 \mathrm{f}$. 
dargelegt hatte, dass das Verbot nicht vom Schutz des Art. 79 Abs. 3 GG umfasst war, war der Weg für den Gerichtshof frei, den Gleichbehandlungsanspruch aus Gründen des Geschlechts ${ }^{19}$ auch gegen jene spezifische Bestimmung des Grundgesetzes durchzusetzen. Noch deutlicher wird die Rücksichtnahme des Gerichtshofes in der Rechtssache „Omega-Spielhallen“. Dort hat er - durchaus überraschend - ein sehr singuläres, spezifisch deutsches Verständnis der Menschenwürde als Rechtfertigung für die Beschränkung einer Dienstleistung (Art. 49 EGV/Art. 56 AEUV) akzeptiert, die überall sonst zulässig ist. Dahinter dürfte letztlich das Damoklesschwert des Art. 1 GG gestanden haben, der bekanntlich vom Schutzbereich der Ewigkeitsgarantie (Art. 79 Abs. 3 GG) umfasst ist und dessen „Berührung“ möglicherweise einen Konflikt zur Folge gehabt hätte, den der Europäische Gerichtshof tunlichst vermeiden wollte.

\section{Ebenenübergreifende Kooperation als Strukturmerkmal der EU}

Ist die Europäische Union unter materiell-rechtlichem Blickwinkel eine wechselseitige Auffangordnung, so ist sie, funktional betrachtet, eine Kooperationsordnung. ${ }^{20}$ Die Erfüllung aller Staatsfunktionen ist in ihr darauf angelegt, dass Einrichtungen der Europäischen Union mit denen der Mitgliedstaaten kooperieren. Wir kennen das aus dem Bereich der Rechtsprechung, wo sowohl der Europäische Gerichtshof in Bezug auf Art. 234 EGV/Art. 267 AEUV $^{21}$ als auch das Bundesverfassungsgericht ${ }^{22}$ ein solches Kooperationsverhältnis postuliert haben. Wir kennen das Kooperationsprinzip auch aus dem Bereich des Vollzugs, ${ }^{23}$ des

19 Richtlinie 76/207/EWG des Rates vom 09.02. 1976 zur Verwirklichung des Grundsatzes der Gleichbehandlung von Männern und Frauen hinsichtlich des Zugangs zur Beschäftigung, zur Berufsbildung und zum beruflichen Aufstieg sowie in bezug auf die Arbeitsbedingungen, ABlEG Nr. L 39/40 v. 14.02. 1976.

20 BVerfG, Vertrag von Lissabon, a. a. O., Rz. 227; in diesem Sinne schon Huber, P. M.: Europäisches und nationales Verfassungsrecht, in: Veröffentlichungen der Vereinigung der Deutschen Staatsrechtslehrer, 60 (2001), 194 ff., hier 209, 228 ff.; ders.: Recht der Europäischen Integration, 2. Aufl., München, 2002, $\S \S 19 \mathrm{ff}$.

21 EuGH, Urt. v. 16. 12. 1981, Rs. 244/80 (Foglia/Novello), Slg. 1981, 3045, hier Rz. 14 ff.; Huber, P. M.: Das Verhältnis des Europäischen Gerichtshofes zu den nationalen Gerichten, in: Merten, D./Papier, H.J. (Hrsg.): Handbuch der Grundrechte in Deutschland und Europa, Bd. VI/2, Heidelberg, 2009, § 172 Rn. 28.

22 BVerfGE 89, 155 ff. (Vertrag von Maastricht), hier 175; in BVerfG, Vertrag von Lissabon, a.a.O., taucht der Begriff des Kooperationsverhältnisses allerdings (bedauerlicherweise) nicht wieder auf.

23 Huber, P. M.: Recht der Europäischen Integration, a. a. O., § 20. 
Europäischen Verwaltungsverbundes, und wir kennen es aus dem Bereich der Rechtssetzung und deren demokratischer Legitimation. ${ }^{24}$

\section{Demokratische Legitimation im Verfassungsverbund}

Rechtssetzung und demokratische Legitimation von Rechtssetzung erfolgen, wenn man so will, in einer Kooperation zwischen dem Europäischen Parlament und den nationalen Parlamenten, die insoweit durch ihre im Rat der Europäischen Union vertretenen Regierungen mediatisiert werden. Den Rat demokratisch zu kontrollieren, ist gemeinsame Aufgabe des Deutschen Bundestags, des tschechischen Parlaments, der Houses of Parliament, der Abgeordnetenkammer in Rom etc., nicht aber des Europäischen Parlaments.

Wenn man sich die Verfassungen der 27 Mitgliedstaaten - einschließlich der ungeschriebenen Verfassung Großbritanniens - ansieht, dann finden sich mehr oder weniger weit reichende, jedoch ziemlich komplizierte Regelungen, die sich mit der Mitwirkung der nationalen Parlamente in europäischen Angelegenheiten beschäftigen. Formal am weitesten reichen sie wohl in Österreich, wo der Nationalrat die Regierung auf die Parlamentsposition verpflichten kann und es (theoretisch) keinen Gestaltungsspielraum der Regierung mehr gibt. Aber auch in Deutschland lesen sich die Mitwirkungsrechte von Bundestag und Bundesrat nach dem Wortlaut des Grundgesetzes und der Beteiligungsgesetze eindrucksvoll, auch wenn der Blick in die Praxis Ernüchterungen bereit hält.

Das Europaverfassungsrecht ${ }^{25}$ setzt jedenfalls auf eine Kooperation von nationalen Parlamenten und Europäischem Parlament, und das gilt auch für das Unionsrecht, das ihnen mit dem Protokoll über die Rolle der einzelstaatlichen Parlamente in der Europäischen Union eine eigenständige Rolle zuweist. Diese Rolle wird mit dem Inkrafttreten des Vertrages von Lissabon durch die in Art. 12 EUV-Lissabon enthaltene Regelung über die Nationalen Parlamente sogar eine spezifische primärrechtliche Verankerung erfahren und mit der Einführung des sog. Frühwarnmechanismus ${ }^{26}$ weiter ausgebaut werden. Damit wird auch der Beitrag der nationalen Parlamente zur demokratischen Legitimation der EU ausdrücklich thematisiert.

24 Ebd., § 19.

25 Zum Begriff Häberle, P.: Gemeineuropäisches Verfassungsrecht und „Verfassung“ der EG, in: Schwarze, J. (Hg.): Verfassungsrecht und Verfassungsgerichtsbarkeit im Zeichen Europas, Baden-Baden, 1998, $11-44$, hier $18 \mathrm{f}$.

26 Art. 6 f. Prot. über die Anwendung der Grundsätze der Subsidiarität und der Verhältnismäßigkeit. 


\section{Zusammenspiel von Europäischem Parlament und nationalen Parlamenten}

Was man bislang nur einer Gesamtschau der Art. 48, 49 EUV, Art. 22 EGV (Art. 25 AEUV) etc. entnehmen konnte, fasst der Vertrag von Lissabon in Art. 10 EUV-Lissabon nun ausdrücklich zusammen: Demokratische Legitimation erfahren die Europäische Union und ihre Entscheidungen sowohl durch das Europäische Parlament als auch durch die nationalen Parlamente. Erst das Zusammenwirken beider vermag die europäische öffentliche Gewalt zu legitimieren. ${ }^{27}$

Das ist letztlich eine überzeugende Bestätigung des viel gescholtenen Maastricht-Urteils. ${ }^{28}$ Denn im Maastricht-Urteil hatte das Bundesverfassungsgericht aus der Perspektive des nationalen Demokratieprinzips festgestellt, dass durch all die Hoheitsrechte, die nach Brüssel übertragen worden sind, durch die zahlreichen Mehrheitsentscheidungen, die im Rat getroffen werden, die demokratische Legitimationsvermittlung des in Deutschland zur Anwendung kommenden Unionsrechts nicht mehr ausschließlich über den Deutschen Bundestag erfolgen kann, sondern der „Legitimationsabstützung“ durch das Europäische Parlament bedarf. Das Gericht hat damit das Bild eines baufälligen Hauses gezeichnet, das durch Pfosten abgestützt werden muss. Und in der Tat bedarf das aus der Perspektive des deutschen Verfassungsrechts zunehmend fragile Gebäude des demokratischen Nationalstaats insoweit einer Absicherung durch das Europäische Parlament.

Mutatis muntandis gilt diese Perspektive auch umgekehrt. Wo die nationale Legitimationsvermittlung über die Kontrolle der im Rat vertretenen Regierung wegen der Anwendung des Mehrheitsprinzips nicht mehr funktioniert, hat der Vertragsgeber dem Europäischen Parlament Mitentscheidungsrechte eingeräumt. Schon im Verfassungskonvent hat diese Konnexität eine zentrale Rolle gespielt: Mehrheitsentscheidungen im Rat und Mitentscheidungsrechte des Europäischen Parlaments sind zwei Seiten einer Medaille. Dem liegt die Vorstellung sich wechselseitig ergänzender Legitimationsquellen zugrunde. Das ermöglicht ein großes Maß an Flexibilität: Geht man davon aus, dass Entscheidungen der unionalen öffentlichen Gewalt bzw. der nationalen öffentlichen Gewalt auf unionsrechtlicher Grundlage auf einem hinreichenden demokratischen Legitimationsni-

27 Huber, P. M.: Recht der Europäischen Integration, a. a. O., § 5 Rn. 41.

28 Bedauerlicherweise wird dieser Faden im Lissabon-Urteil des BVerfG nicht fortgeführt. 
veau beruhen müssen, dann muss - wenn der Beitrag einer Seite zu gering ausfällt - dies auf der anderen Seite kompensiert werden.

\section{Begrenzte Legitimationskraft des Europäischen Parlaments}

Betrachtet man vor diesem Hintergrund noch einmal den Beitrag, den das Europäische Parlament zur Legitimation der EU leistet, dann stellt man fest, dass die Europawahlen wichtige Funktionen nicht erfüllen, dass sie insbesondere keine real- und personalplebiszitäre Grundentscheidung der Bürger darstellen. Während Wahlen im nationalen Kontext idealtypisch durch die politischen Parteien vorstrukturiert werden, während die politische Richtungsbestimmung durch Wahlen seit 60 Jahren mehr oder weniger funktioniert und der Bruch von Wahlversprechen politisch durchaus sanktioniert wird ${ }^{29}$ - die Vorgänge um die Regierungsbildung in Hessen 2008/09 haben dies erst wieder eindrucksvoll bestätigt kann davon auf der Ebenen der Europäischen Union keine Rede sein. Seit 1979 kann kein Wähler feststellen, ob seine Stimmabgabe irgendeinen Effekt gehabt hat, und wenn ja, welchen. Angesichts der fraktionsübergreifenden ,großen“ Koalitionen, die es im Europäischen Parlament von Anfang an gab, und angesichts des Fehlens einer Opposition erfüllt die Wahl zur Europäischen Parlament die wesentliche Funktion der Legitimationsvermittlung bis heute nicht.

Das beruht ganz entscheidend darauf, dass es an politischen Parteien fehlt, die den Willensbildungsprozess im Vorfeld der Europawahlen strukturieren und alternative Politikkonzepte herausbilden könnten. Solange die Europawahlen für die nationalen politischen Parteien vor allem ein Instrument zur Verbesserung der Parteienfinanzierung sind, solange sie die Wahlen - ähnlich wie die Landtagswahlen $^{30}$ - auch bzw. vor allem als Testwahl für Berlin verstehen, für Paris oder London - solange hält sich der Beitrag des Europäischen Parlaments zur demokratischen Legitimation der EU in engen Grenzen. Zwar gibt es seit ein paar Jahren ein europäisches Parteienstatut; ${ }^{31}$ dieses regelt aber bedauerlicherweise in erster Linie, wie man öffentliche Mittel auf die europäischen Parteienverbünde verteilt und weniger, wie die politischen Parteien auf europäischer

29 Huber, P.M.: Der Parteienstaat als Kern des politischen Systems, Juristenzeitung, 49/14 (1994), 689696.

30 Ders.: Deutschland in der Föderalismusfalle?, Heidelberg, 2003.

31 Verordnung (EG) Nr. 2004/2003 des Europäischen Parlaments und des Rates vom 04. 11. 2003 über die Regelungen für die politischen Parteien auf europäischer Ebene und ihre Finanzierung, ABIEU Nr. L 297/1 v. 15. 11. 2003; Kluth, W., in: Calliess, C./Ruffert, M. (Hg.): EUV/EGV, 3. Aufl., München, 2007, Art. $191 \mathrm{Rn} .6 \mathrm{ff}$. 
Ebene tatsächlich zur Herausbildung eines europäischen politischen Bewusstseins und zur Artikulation des Willens der Bürgerinnen und Bürger beitragen können (Art. 191 EGV/Art. 10 Abs. 4 EUV-Lissabon, Art. 224 AEUV). Vor diesem Hintergrund ist der Beitrag, den das Europäische Parlament zur demokratischen Legitimation der EU zu leisten vermag, ungeachtet seiner Kompetenzen, nach wie vor gering, und das gilt - wie die geringe Wahlbeteiligung bei den Europawahlen zeigt - auch für deren Integrationskraft.

\section{Exkurs}

Freilich - das sei nebenbei bemerkt - hat die eher unpolitische, technokratische Ausrichtung des Europäischen Parlaments auch Vorteile. Verglichen mit den Parlamenten auf nationaler und regionaler Ebene, insbesondere mit dem Deutschen Bundestag und den Landtagen, erscheint die Arbeit im Europäischen Parlament geradezu als Idealfall konzentrierter Sachlichkeit. Während man sich auf nationaler Ebene mitunter an das Bismarck-Bonmot erinnert fühlt, dass es besser sei, wenn die Leute nicht wissen, wie Würste und Gesetze gemacht werden, schaffen es im Europäischen Parlament mehr als 100 Abgeordnete und andere Beteiligte regelmäßig, einen oder mehrere Tage konzentriert, sachorientiert und ohne polemische Schärfe um das sachliche Für und Wider einer Regelung zu ringen. Insoweit gleicht das Europäische Parlament dem House of Lords, das sich - entrückt von den Rationalitäten parteipolitischer Mehrheitsbildung - ebenfalls durch eine fach- und sachorientierte Arbeit auszeichnet. Wenn parteipolitische Rationalitäten in die Quere kommen, ist es mit der Sachlichkeit dagegen aus. Diese parteipolitischen Rationalitäten, das Ringen um Machtgewinn und Machterhalt, aber sind das Proprium der Demokratie.

\section{Folgerungen}

Was folgt aus alldem? Einen höheren Beitrag des Europäischen Parlaments zur demokratischen Legitimation der EU kann man nicht dekretieren. Die rechtlichen Voraussetzungen und Verfahren sind zwar vorhanden; dass sie mit Leben gefüllt werden, hängt jedoch an einer Fülle vor allem vorrechtlicher Voraussetzungen. Helmut Schmidt hat kürzlich auf einer Veranstaltung in Berlin geschildert, wie er 1976 mit Giscard d'Estaing die Direktwahl des Europäischen Parlaments auf dem Weg gebracht hat. Dabei seien sie - um ein anderes BismarckWort abzuwandeln - davon ausgegangen, dass das Europäische Parlament, wenn 
es denn einmal aufs Pferd gesetzt worden sei, schon werde reiten können. Nach 30 Jahren müsse er freilich feststellen, dass dies nicht so richtig geklappt habe.

Vor diesem Hintergrund ist es das Gebot der Stunde, die europäische Rolle der nationalen Parlamente weiter zu stärken. Der Vertrag von Lissabon tut dies auf europarechtlicher Ebene (Art. 12 EUV-Lissabon, Art. 1 ff. Protokoll über die Rolle der nationalen Parlamente in der Europäischen Union; Art. 1 ff. Protokoll über die Anwendung der Grundsätze der Subsidiarität und der Verhältnismäßigkeit), und das Bundesverfassungsgericht hat dies im nationalen Kontext für den Deutschen Bundestag zu erzwingen versucht. Dem ist der Gesetzgeber umgehend - wenn auch zähneknirschend - gefolgt. Gleichwohl bleibt die Frage, ob diese mittlerweile sehr elaborierten Regelungen das Papier wirklich wert sind, auf das sie gedruckt sind, oder ob sie ,, law in the books " bleiben werden. Denn der eigentliche Grund, warum die spätestens seit den 1980er Jahren kontinuierlich ausgeweitete Kontrolle der Bundesregierungen im Rat nicht wirklich funktioniert, sind nicht die Regelungen über die Zusammenarbeit in europäischen Angelegenheiten, sondern die Rationalitäten des Parteienstaates. Die angesichts des Grundsatzes der Spiegelbildlichkeit dem Plenum entsprechenden Mehrheiten im Europaausschuss wie in den Fachausschüssen haben i.d.R. kein Interesse daran, der ,eigenen“ Regierung gegen das Schienbein zu treten. Entsprechend lustlos erfolgt die Befassung mit unionsrechtlichen Vorlagen. Das kann jedoch nicht das letzte Wort sein.

Wenn die Mitgliedstaaten „Herren der Verträge“ sind, und wenn die Parlamente der Mitgliedstaaten die vom Europäischen Parlament bis heute (noch) nicht gefüllte Lücke schließen müssen, dann müssen sie ihre europäische Berufung annehmen und daraus Konsequenzen ziehen. Sie müssen ihre Arbeitsweise verändern und begreifen, dass es für eine effektive Politikgestaltung mehr denn je darauf ankommt, unionale Rechtsakte zu initiieren, sie zu begleiten und zu beeinflussen. Daher sollte man die Zusammensetzung der Bundestagsausschüsse an die Zusammensetzung des Rats anlehnen, den Sitzungskalender an Brüssel orientieren und sich auf den Zeitdruck einrichten, unter dem europäische Entscheidungen fallen. Nach wie vor überzeugend wirkt hier der Vorschlag von Rupert Scholz, einen Gemeinsamen Ausschuss von Bundestag und Bundesrat einzusetzen, der ad hoc reagieren und dem deutschen Vertreter Zustimmung oder Verweigerung signalisieren kann, so wie es der Marktausschuss des dänischen Folketing seit Jahren erfolgreich praktiziert - wenn auch unter der Konstellation einer Minderheitsregierung. Man sollte, um einen weiteren Punkt zu nennen, die Grün- und Weißbücher sowie die zahllosen Rechtssetzungsinitiativen im Bun- 
destag systematisch sichten, bewerten und priorisieren, damit das Parlament in der Lage ist, die richtigen Akzente zu setzen.

Langfristig ist die Europäische Union allerdings so offen und flexibel angelegt, dass das Europäische Parlament auch einen erheblich größeren Beitrag zu ihrer demokratischen Legitimation leisten kann, als dies heute der Fall ist - wenn die Voraussetzungen denn stimmen. Das setzt insbesondere politische Parteien auf unionaler Ebene voraus, die mehr sind als Dachorganisationen ihrer nationalen Mitgliedsorganisationen. Ohne einen substantiellen Machtverzicht bei den nationalen Parteizentralen wird dies hingegen kaum realisierbar sein. Bis dahin sollten wir auf das Bewährte vertrauen: auf die Legitimation der Europäischen Union auch und zuvörderst durch unsere nationalen Parlamente. 